\title{
Mass-radius relation of low and very low-mass stars revisited with the VLTI ${ }^{\star}$
}

\author{
B.-O. Demory ${ }^{1}$, D. Ségransan ${ }^{1}$, T. Forveille ${ }^{2}$, D. Queloz ${ }^{1}$, J.-L. Beuzit ${ }^{2}$, X. Delfosse ${ }^{2}$, E. Di Folco ${ }^{1,3}$, P. Kervella ${ }^{3}$, \\ J.-B. Le Bouquin ${ }^{4}$, C. Perrier ${ }^{2}$, M. Benisty ${ }^{5}$, G. Duvert ${ }^{2}$, K.-H. Hofmann ${ }^{6}$, B. Lopez ${ }^{7}$, and R. Petrov ${ }^{8}$ \\ 1 Observatoire de Genève, Université de Genève, 51 chemin des Maillettes, 1290 Sauverny, Suisse \\ e-mail: Brice-0livier.Demory@unige.ch \\ 2 Laboratoire d'Astrophysique de Grenoble, 414 rue de la piscine, Domaine Universitaire de Saint Martin d'Hères, \\ 38041 Grenoble, France \\ 3 LESIA, Observatoire de Paris, CNRS UMR 8109, UPMC, Université Paris Diderot, 5 place Jules Janssen, 92195 Meudon, France \\ 4 European Southern Observatory, Casilla 19001, Vitacura, Santiago 19, Chile \\ 5 INAF-Osservatorio Astrofisico di Arcetri, Largo Fermi 5, 50125 Firenze, Italy \\ 6 Max-Planck-Institut für Radioastronomie, Auf dem Hügel 69, 53121 Bonn, Germany \\ 7 Laboratoire Fizeau, UMR 6525 Observatoire de la Côte d'Azur, Boulevard de l'Observatoire, BP 4229, \\ 06304 Nice Cedex 4, France \\ ${ }^{8}$ Laboratoire Fizeau, UMR 6525 Université de Nice-Sophia Antipolis, Parc Valrose, 06108 Nice Cedex 02, France
}

Received 2 March 2009 / Accepted 16 May 2009

\section{ABSTRACT}

\begin{abstract}
We measured the radii of 7 low and very low-mass stars using long baseline interferometry with the VLTI interferometer and its VINCI and AMBER near-infrared recombiners. We use these new data, together with literature measurements, to examine the luminosityradius and mass-radius relations for $\mathrm{K}$ and $\mathrm{M}$ dwarfs. The precision of the new interferometric radii now competes with what can be obtained for double-lined eclipsing binaries. Interferometry provides access to much less active stars, as well as to stars with much better measured distances and luminosities, and therefore complements the information obtained from eclipsing systems. The radii of magnetically quiet late- $\mathrm{K}$ to $\mathrm{M}$ dwarfs match the predictions of stellar evolution models very well, providing direct confirmation that magnetic activity explains the discrepancy that was recently found for magnetically active eclipsing systems. The radii of the early $\mathrm{K}$ dwarfs are reproduced well for a mixing length parameter that approaches the solar value, as qualitatively expected.
\end{abstract}

Key words. stars: low-mass, brown dwarfs - stars: fundamental parameters - techniques: interferometric

\section{Introduction}

Measuring accurate masses, radii, and luminosities for low and very low-mass stars has always been observationally challenging. Precise individual masses and radii can be measured through combined photometric and spectroscopic observations of double-lined eclipsing binaries, but to date only $\sim 15$ such systems are known with masses under 1 solar mass, and a few of those are too distant and faint for high-precision work. Perhaps even more important, low-mass eclipsing binaries tend, almost by construction, to be fast rotators, hence magnetically very active. Evolutionary models have been found to systematically underestimate the radii of very low-mass eclipsing binaries (Torres $\&$ Ribas 2002), and the uniformally high activity level of these objects is currently the leading explanation for that discrepancy. Chabrier et al. (2007) find that increased surface-spot coverage and, for partly convective stars, convection quenching by strong magnetic fields can inflate the stellar radius by amounts that qualitatively match the observed discrepancy.

Direct tests of that prediction and validation of the 1-D structural models on objects that better match their assumptions need

* Based on data collected with the VLTI/VINCI and VLTI/AMBER instruments at ESO Paranal Observatory, programmes ID 60.A-9220, 080.D-0653 and 082.D-0196. radius measurements for slowly rotating and magnetically quiet very low-mass stars. Strong observational selection effects unfortunately ensure that all known eclipsing binaries have short enough orbital periods to be tidally synchronised, hence fast rotators. Measurements of magnetically quiet slow rotators therefore have to use a different observing technique, either longbaseline optical or infrared interferometry. Lane et al. (2001) and Ségransan et al. (2003) both demonstrate $1-5 \%$ radius precision for stars that are only partially resolved.

The mass of these single, isolated stars is not directly accessible, and, strictly speaking, both Lane et al. (2001) and Ségransan et al. (2003) therefore probe the luminosity-radius relation rather than the mass-radius one. Accurate mass and luminosity measurements for $\mathbf{M}$ dwarfs (e.g. Ségransan et al. 2000), however, demonstrate that their $K$-band mass-luminosity relation has very low dispersion (Delfosse et al. 2000), and mass is therefore largely interchangeable with absolute $K$-band luminosity.

Here we present direct angular diameter measurements for seven single K0.5 to M5.5 dwarfs, obtained with the VINCI and AMBER instruments on the Very Large Telescope Interferometer (VLTI) between 2003 and 2008. Section 2 describes those observations, the data analysis, and the angular diameter determination. Section 3 discusses the luminosity-radius 
Table 1. Observation log of stars published in this work.

\begin{tabular}{|c|c|c|c|c|c|c|c|}
\hline$\overline{\text { Star }}$ & $\begin{array}{l}\text { Spectral } \\
\text { Type }\end{array}$ & $\overline{m_{K}}$ & Instrument & $\overline{\text { Date }}$ & Baseline & $\begin{array}{c}\text { DIMM Seeing } \\
{\left[{ }^{\prime \prime}\right]} \\
\end{array}$ & $\begin{array}{c}\text { Mean } \tau_{0} \\
{[\mathrm{~ms}]}\end{array}$ \\
\hline \multirow[t]{4}{*}{ GJ 663 A } & $\mathrm{K} 0 \mathrm{~V}$ & - & VINCI & $13-05-2003$ & B3-M0 (140 m.) & - & - \\
\hline & & & & $26-05-2003$ & B3-M0 (140 m.) & 0.7 & 5 \\
\hline & & & & $27-05-2003$ & B3-M0 (140 m.) & 0.5 & 7 \\
\hline & & & & $28-05-2003$ & B3-M0 (140 m.) & 0.8 & 6 \\
\hline GJ 166 A & $\mathrm{K} 0.5 \mathrm{~V}$ & 2.41 & AMBER & $27-10-2007$ & A0-K0-G1 & 1.2 & 3 \\
\hline \multirow[t]{10}{*}{ GJ $570 \mathrm{~A}$} & $\mathrm{~K} 4 \mathrm{~V}$ & 3.06 & VINCI & $07-04-2003$ & B3-M0 (140 m.) & 0.6 & 4 \\
\hline & & & & 08-04-2003 & B3-M0 (140 m.) & 0.5 & 6 \\
\hline & & & & $12-04-2003$ & B3-M0 (140 m.) & 0.7 & 6 \\
\hline & & & & $15-04-2003$ & B3-M0 (140 m.) & 0.5 & 9 \\
\hline & & & & $16-04-2003$ & B3-M0 (140 m.) & 0.7 & 5 \\
\hline & & & & 21-04-2003 & B3-M0 (140 m.) & 0.7 & 4 \\
\hline & & & & 27-04-2003 & B3-M0 (140 m.) & 0.5 & 3 \\
\hline & & & & $07-05-2003$ & B3-M0 (140 m.) & 1.2 & 2 \\
\hline & & & & 08-05-2003 & B3-M0 (140 m.) & 1.2 & 3 \\
\hline & & & & 09-05-2003 & B3-M0 (140 m.) & 0.6 & 7 \\
\hline \multirow[t]{10}{*}{ GJ 845 A } & K5V & 2.18 & VINCI & $15-09-2002$ & E0-G1 (66 m.) & 1.1 & 2 \\
\hline & & & & $16-09-2002$ & E0-G1 (66 m.) & 1.0 & 2 \\
\hline & & & & 17-09-2002 & E0-G1 (66 m.) & 0.8 & 2 \\
\hline & & & & 10-10-2002 & B3-M0 (140 m.) & 0.9 & 2 \\
\hline & & & & $12-10-2002$ & B3-M0 (140 m.) & 1.1 & 4 \\
\hline & & & & $16-10-2002$ & B3-M0 (140 m.) & 0.8 & 7 \\
\hline & & & & $17-10-2002$ & B3-M0 (140 m.) & 1.2 & 5 \\
\hline & & & & $19-10-2002$ & B3-M0 (140 m.) & 0.6 & 4 \\
\hline & & & & $22-10-2002$ & B3-M0 (140 m.) & - & - \\
\hline & & & & 26-10-2002 & B3-M0 (140 m.) & 0.9 & 3 \\
\hline GJ 879 & $\mathrm{~K} 5 \mathrm{Vp}$ & 3.81 & AMBER & $03-10-2008$ & $\mathrm{~A} 0-\mathrm{K} 0-\mathrm{G} 1$ & 1.4 & 1 \\
\hline GJ 887 & $\mathrm{M} 0.5 \mathrm{~V}$ & 3.36 & AMBER & $27-10-2007$ & A0-K0-G1 & 1.2 & 3 \\
\hline GJ 551 & M5.5V & 4.38 & AMBER & $27-02-2008$ & $\mathrm{~A} 0-\mathrm{K} 0-\mathrm{G} 1$ & 1.0 & 3 \\
\hline
\end{tabular}

and mass-radius relation for very low-mass stars in the light of the new measurements and compares the empirical relations with theoretical predictions.

\section{Observations and data analysis}

\subsection{Sample}

The target list was largely determined by the capabilities of the VLTI at the time of the observations. The limiting correlated magnitude of the low spectral resolution mode of AMBER on the $1.8 \mathrm{~m}$ auxiliary telescopes was $K=4$ in 2007, and improved to $K=5.5$ in 2008 . We consequently selected targets with apparent magnitudes between $K=2.18$ and $K=4.38$. Ongoing improvements to the VLTI infrastucture are expected to provide access to fainter targets. We also computed the expected angular diameters from flux-colour relations and literature photometry, and selected targets with a predicted diameter above 0.9 mas. This translates into a visibility of at most 0.8 on a $128 \mathrm{~m}$ baseline in the $H$-band, as needed to measure diameters to a few $\%$ uncertainty with the current amplitude calibration precision of AMBER. One object, GJ 879, was observed as a backup target during an unrelated observing programme, and does not fulfil this minimum angular diameter specification.

\subsection{Observations}

\subsubsection{VINCl observations}

GJ 845 A ( $\epsilon$ Ind), GJ 166 A (DY Eri), GJ 570 A (KX Lib), and GJ 663 A were observed on the ESO Very Large Telescope Interferometer (VLTI) using its commissioning instrument, VINCI (Kervella et al. 2000) with the two $35 \mathrm{~cm}$ test siderostats.
Table 1 summarises the observation details. VINCI operated in the $K$-band and used single-mode optical fiber couplers to recombine the light from two telescopes, and modulated the optical path difference around the white-light fringe to produce interference fringes. This recombination scheme, first used in the FLUOR instrument (Coude Du Foresto et al. 1998), produces high-precision visibility values, thanks to the efficient conditioning of the incoming wavefronts by the single mode fibers, to photometric monitoring of the light coupled into each input fibers, and to fast scanning of the high-quality fringes. Kervella et al. (2004a) extensively describe the data reduction for VINCI.

Our observing strategy alternated sequences of several hundred fringe scans on the target star and on spatially close calibrator stars, to efficiently sample the temporal and spatial structure of the atmospheric and instrumental transfer function. Adherence to this strategy could unfortunately not always be strict, since scientific observations often had to give way to VLTI commissioning activities. For GJ $166 \mathrm{~A}$, in particular, we could only keep three data points since all other measurements had no acceptably close calibrator observations. That target was also observed under poorer atmospheric conditions than all other sources, and with two calibrators located 78 and 113 degrees away. It is by far our worst quality measurement, so is discarded from this study.

\subsubsection{AMBER observations}

We used the AMBER (Astronomical Multi-BEam combineR) recombiner of the VLTI to measure the radiii of GJ $166 \mathrm{~A}$, GJ 887, Proxima (GJ 551), and GJ 879. Table 1 summarises the observing circumstances. AMBER uses single-mode fibers for 
wavefront filtering and produces spectrally-dispersed fringes in the $J, H$, and $K$ near-infrared bands (Petrov et al. 2007).

We used the $1.8 \mathrm{~m}$-diameter VLT auxilliary telescopes (AT) on the A0-K0-G1 baseline triplet, which at the time of our observations offered the longest available baselines and therefore the highest angular resolution. We selected the low spectral resolution mode (low-JHK) of AMBER, which covers the $J, H$, and $K$ bands with $R=30$, and adopted a $50 \mathrm{~ms}$ exposure time. AMBER unfortunately has poor $J$-band sensitivity, and we detected no fringes in that band. The $H$-band fringes, on the other hand, probe significantly higher spatial frequencies than the $K$-band VINCI would have on the same baselines. We mostly chose not to use the FINITO fringe tracker (Le Bouquin et al. 2008), since our targets were at best close to the $H=3$ limiting magnitude of FINITO. The fringes would thus not have been stabilized enough to allow much longer integration times and compensate for the $80 \%$ FINITO levy on the $H$-band flux.

Since accurate absolute visibility calibration with AMBER had not been demonstrated when we planned the observations, we requested a very conservative observing strategy. Observations of up to four different amplitude calibrators were interleaved with those of each science target, to closely monitor the instrumental and atmospheric transfer function. Those calibrators were chosen from Merand et al. (2004) for angular proximity, well-constrained predicted visibilities, and an approximate magnitude and colour match to the science targets. The last requirement had to be relaxed somewhat for the $M$ dwarf targets, since we could locate no appropriate M-type calibrators. For those stars, we thus used K giant calibrators, which remain fairly close in near-IR colours. Since AMBER had never been used to measure precise angular radii, we chose to observe two stars again that had been previously measured with VINCI, GJ 551, and GJ 887. VINCI has a well-established record of accurate amplitude calibration, and those three stars provide a valuable check on any potential systematics in the AMBER measurements.

GJ 879 was observed as a backup target in very poor atmospheric conditions (seeing $F W H M>1.5^{\prime \prime}$ and $\tau_{0}<2 \mathrm{~ms}$ ), and has the smallest angular radius in our sample (highest $V^{2}$ ). Its radius measurement, as a consequence, has significantly larger error bars than any other AMBER source.

\subsection{Data reduction}

With angular sizes under 2 mas, our targets are only partially resolved on the longest baselines available for our observations ( $128 \mathrm{~m}$ on the A0-K0 baseline with AMBER, and $140 \mathrm{~m}$ on the B3-M0 baseline with VINCI). Their squared visibilites $V^{2}$ remain above 0.5 in the $H$-band. We therefore cannot derive their angular diameters from just the location of the first null of the visibility function, and instead need accurate calibration of the visibilities. The very partial resolution, on the other hand, ensures that bandwidth-smearing effects are negligible. At our precision level on the visibilities, accounting for the finite spectral bandwidth would only become necessary for $V^{2}$ below 0.3 for VINCI, and well beyond the first visibility null for AMBER.

The end products of both the VINCI and the AMBER pipelines consist of coherence factors $\left(\mu^{2}\right)$, together with an internal error estimate on that quantity. The coherence factor is related to the squared visibility, $V^{2}$, through

$V_{\lambda}^{2}=\frac{\mu_{\lambda}^{2}}{T_{\lambda}^{2}}$,
Table 2. List of calibrator stars used during VINCI runs.

\begin{tabular}{|c|c|c|c|c|c|}
\hline Calibrator & "Target & $\begin{array}{c}\text { Ang. dist. } \\
\text { degrees }\end{array}$ & $\begin{array}{l}\text { Spectral } \\
\text { Type }\end{array}$ & $\bar{m}_{K}$ & $\begin{array}{c}\theta_{\mathrm{UD}} K \text { band } \\
{[\mathrm{mas}]}\end{array}$ \\
\hline HR & & $78.3,19.3$ & MOIII & 1.98 & 2.01 \\
\hline$\delta \mathrm{Ph}$ & $\mathrm{G}$ & & G9III & 1. & .02 \\
\hline HP & GJ & 12 & M0III & & 2 \\
\hline HD 1 & GJ & 2.4 & K5III & 2.10 & $2.04=$ \\
\hline$\chi \mathrm{Sco}$ & GJ 570 A, GJ 663 A & $20.6,20.7$ & K3III & 2.09 & $2.04 \pm 0.02$ \\
\hline
\end{tabular}

References: angular diameters come from Bordé et al. (2002) and Merand et al. (2004).

Table 3. List of calibrator stars used during AMBER runs.

\begin{tabular}{llcllll}
\hline \hline Calibrator & Target & $\begin{array}{c}\text { Ang. dist. Spectral } \\
\text { degrees }\end{array}$ & $\begin{array}{l}m_{K} \\
\text { Type }\end{array}$ & $\begin{array}{c}\theta_{\text {UD }} K \text { band } \\
{[\mathrm{mas}]}\end{array}$ \\
\hline HD 25700 & GJ 166 A & 9.3 & K3III & 3.16 & $1.04 \pm 0.01$ \\
HD 27508 & GJ 166 A & 9.9 & K5III & 3.60 & $0.98 \pm 0.01$ \\
HD 127897 & GJ 551 & 10.2 & K4III & 3.82 & $0.91 \pm 0.01$ \\
HD 128713 & GJ 551 & 6.4 & K0.5II & 3.49 & $0.86 \pm 0.01$ \\
HD 130227 & GJ 551 & 6.5 & K1III & 3.59 & $0.92 \pm 0.01$ \\
HD 136289 & GJ 551 & 10.1 & K3III & 3.54 & $0.94 \pm 0.01$ \\
HD 204609 GJ 879 & 19.9 & K7III & 3.20 & $1.14 \pm 0.02$ \\
HD 205096 GJ 879 & 19.8 & K1III & 3.82 & $0.82 \pm 0.01$ \\
HD 215627 & GJ 879, GJ 887 & $10.3,6.9$ & K2III & 3.91 & $0.83 \pm 0.01$ \\
HD 221370 GJ 887 & 7.8 & K2III & 3.61 & $0.90 \pm 0.01$ \\
HD 223428 & GJ 879 & 12.4 & K1III & 3.21 & $1.07 \pm 0.01$ \\
\hline
\end{tabular}

References: angular diameters come from Merand et al. (2004).

where $T^{2}$ is the squared transfer function of the instrument plus the atmosphere.

Accurate calibration of the absolute squared visibilities therefore critically depends on a well-understood transfer function. That function is sensitive to both instrument stability and atmospheric conditions, and can fluctuate during a night. To assess its stability, we calculated the transfer function for every calibrator exposure during a night, disregarding only those datasets for which too few scans/frames passed the reduction pipeline's thresholds to ensure the statistical significance of the resulting coherence factor measurement. In most cases, the transfer function for each target measurement was evaluated from two different calibrators, providing some control for temporarily deteriorated atmospheric conditions. Using two calibrators also protects against systematics introduced by a poorly chosen calibrator, such as unrecognised binaries. When no calibrator was observed immediately before or after a target point, we adopted the mean of the transfer function for the two nearest calibrators, provided they were observed within 1 h of the science measurement. A few observations had to be discarded because no sufficiently close pair of calibrator observations was available. Tables 2 and 3 respectively summarise the calibrators' properties for the AMBER and the VINCI observations.

The contribution of the calibration to the visibility error bars accounts for both the statistical uncertainty on the coherence factor and the propagation of the uncertainty on the calibrators' diameters. Since the different $V^{2}$ measurements for a given target share any systematic error on the calibrators' diameters, they cannot be considered as fully independent. Those correlations are accounted for in the error bars, using the method described by Perrin (2003). That method is directly applicable to VINCI observations, but needed adaptation for AMBER, as we explain later. 


\subsubsection{VINCI data reduction}

We used V. 3.1 of the standard VINCI reduction pipeline (Kervella et al. 2004a). We used the wavelet spectral density (Ségransan et al. 1999) as a visibility estimator, because we found that it removes pistoned interferograms more robustly than the more common Fourier analysis. The commissioning state of the instrument and of the VLTI array often affected the observing strategy, so we had to discard a significant number of measurements for which no calibrator observations where recorded at the same scan frequency. Kervella et al. (2004a) showed, from observations of brighter calibrators, that the squared transfer function of VINCI instrument is stable to within $1.5 \%$ during a night. We could therefore average the transfer function measurements from all calibrators observed during one night, and the observed dispersion mainly reflects the statistical errors on these individual measurements. Those are the main source of uncertainties on our final radii obtained with VINCI.

\subsubsection{AMBER data reduction}

General description We used the AMBER data reduction pipeline described by Tatulli et al. (2007). After data reduction, we noted structures in the $V^{2}$ data obviously resulting from correlations in the dataset presumably due to non optimal pipeline settings. Indeed, in February 2008, the AMBER Task Force team (Malbet et al. 2008) reported the displacement of the photometric channels with respect to the interferometric channel between 2004 and 2008. Correlations occur on several pixels if those displacements are not correctly calibrated, a step that is achieved through the AMBER standard calibration matrix computation. During our October 2007 observing run, the spectrograph entry slit was tilted and badly focused, thus propagating correlations over 4 to 5 pixels on the detector, while AMBER spectral resolution is usually sampled over 2 pixels. We corrected this effect by taking integer pixel channel offsets into account during spectral reshifting procedure, to avoid additional correlations to appear during the subtraction of the bad-pixel map at the subpixel level. None of our targets had enough $J$-band flux to offset the poor transmission of AMBER in that band. We therefore discarded the $J$-band data, along with wavelengths affected by telluric absorption, only keeping the centres of the $H$ and $K$ bands, $1.65-1.85 \mu \mathrm{m}$ and $2.10-2.40 \mu \mathrm{m}$.

We concatenated all observations of each object (usually 5 sets of 1000 frames) into a single dataset from which we could more robustly select on fringe SNR, to reject pistoned interferograms, as well as scans with low flux on one of the two telescopes in a pair. We verified that selecting the best $20 \%$ to $75 \%$ frames produced similar results, hence that the details of the selection do not unduly affect the outcome. Our final was to keep the best $20 \%$, as a trade-off between fringe jitter suppression and increased noise. Selection based on an absolute SNR threshold would reject blurred fringes (which bias down $V^{2}$ ) more effectively than accepting a specified fraction of the data, but that alternate mode is not available in the current version of the AMBER pipeline. Another limitation of that data reduction package is that it uses a common threshold for all 3 baselines, in spite of their quite different throughputs. Those limitations would become more critical for datasets containing less scans than we used here.
27 October 2007
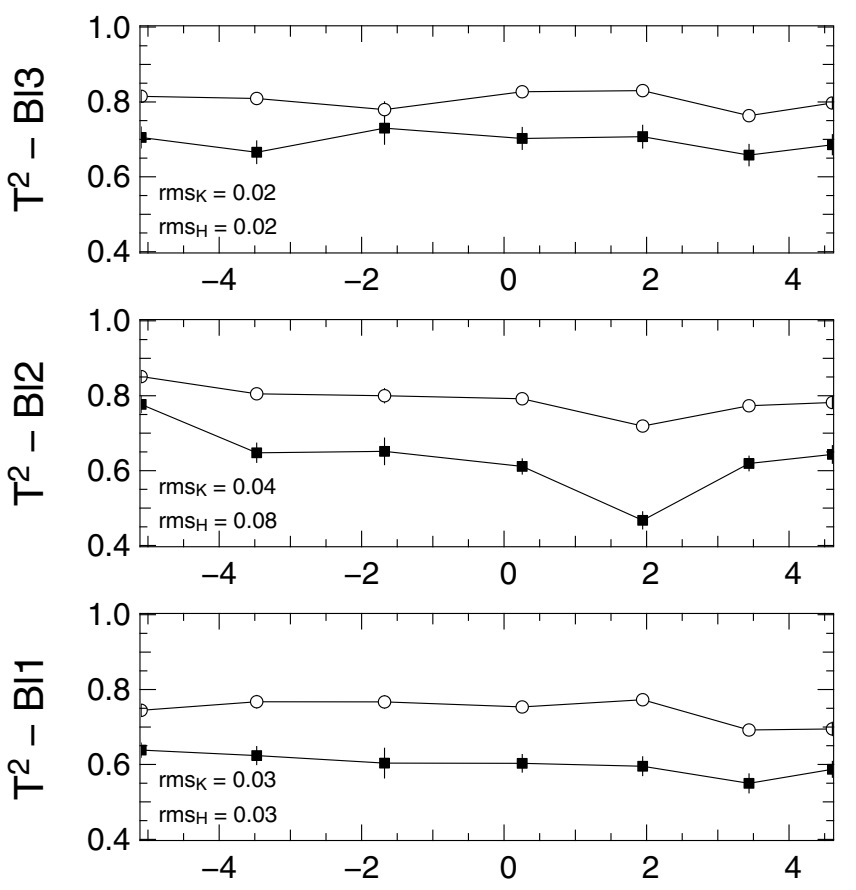

Time $[\mathrm{h}]$

Fig. 1. AMBER squared transfer function for 2007 Oct. 27. The upper, middle and bottom panel respectively represent baselines 3,2 , and 1 . Circles and squares respectively represent the values for the centre wavelengths of the $K$-band $(2.25 \mu \mathrm{m})$ and of the $H$-band $(1.76 \mu \mathrm{m})$, The corresponding rms dispersions are reported in each panel.

Transfer function As discussed above, a well-understood transfer function is critical for measuring absolute visibilities. The stability of the VINCI transfer function is well-established, and a wealth of absolute visibilities have been published with that instrument. The reliability of AMBER for absolute $V^{2}$ measurements, on the other hand, has been questioned and the stability of the AMBER $T^{2}$ needs to be established. Figure 1 shows the squared transfer functions of the 3 AMBER baselines during the night of 2007 October 27. The atmospheric conditions during the first half of the night were representative of Paranal, with a 0.8 arcsec seeing and a coherence time slightly above $3 \mathrm{~ms}$. With a 2 to $4 \%$ rms dispersion for both bands on baselines 1 and 3 (90 and 128 m length respectively) and in $K$-band for baseline $2(90 \mathrm{~m})$, AMBER approaches the stability of the VINCI transfer function. The end of the night had severely degraded atmospheric conditions (2.5 arcsec seeing and $1 \mathrm{~ms}$ coherence time), and the transfer function degraded only moderately, except on baseline 2 , which had shown a $13 \%$ rms dispersion.

Error bar computation The reliability of absolute $V^{2}$ measured with AMBER has not yet been established, so care is needed when evaluating error bars. The 16 to 18 spectral channels that we usually kept ( 7 to 8 in the $H$-band, and 9 to 10 in the $K$-band) were measured simultaneously. They therefore shared the same atmosphere, as well as any error on the angular diameter of the calibrator(s). If those factors dominate statistical noise, the individual channels become highly dependent.

To quantify these correlations between spectral channels, we generalise the formalism developed by Perrin (2003) for a 
Table 4. Stellar properties.

\begin{tabular}{|c|c|c|c|c|c|c|c|c|c|c|c|c|}
\hline$\overline{\text { Star }}$ & $\begin{array}{l}\text { Spect. } \\
\text { Type }\end{array}$ & $\overline{\overline{M_{K}}}$ & $\begin{array}{c}\text { Teff } \\
\mathrm{K} \\
\end{array}$ & $\overline{\overline{\text { Ref. }}}$ & $\begin{array}{l}\text { Mass } \\
{\left[M_{\odot}\right]} \\
\end{array}$ & $\overline{[\overline{\mathrm{Fe}} / \mathrm{H}]}$ & Ref. & $\begin{array}{c}\text { Derived } T_{\text {eff }} \\
\mathrm{K} \\
\end{array}$ & $\begin{array}{c}v \sin i \\
\mathrm{~km} \mathrm{~s}^{-1}\end{array}$ & $\overline{\log R_{H K}^{\prime}}$ & Instr. & $\begin{array}{c}\text { Radius } \\
{\left[R_{\odot}\right]} \\
\end{array}$ \\
\hline GJ 663 A & K0V & - & - & & - & -0.20 & (3) & $4843 \pm 134$ & - & - & VINCI & $0.817 \pm 0.016$ \\
\hline GJ 166 A & $\mathrm{K} 0.5 \mathrm{~V}$ & $90 \pm 0.02$ & 5201 & (3) & $877 \pm 0.044$ & -0.25 & (3) & $9 \pm 35$ & 0.78 & -4.87 & AMBER & 170 \\
\hline GJ $570 \mathrm{~A}$ & K4V & $4.20 \pm 0.03$ & 4758 & (3) & $0.802 \pm 0.040$ & 0.06 & (3) & $4597 \pm 101$ & 1.50 & -4.48 & VINCI & $0.739 \pm 0.019$ \\
\hline GJ 845 & K5V & $4.38 \pm 0.03$ & 4630 & (3) & $0.762 \pm 0.038$ & -0.06 & (3) & $4568 \pm 59$ & 1.46 & -4.56 & VINCI & $0.732 \pm 0.006$ \\
\hline GJ 879 & K5Vp & $4.54 \pm 0.08$ & 4574 & (3) & $0.725 \pm 0.036$ & 0.02 & (3) & $4711 \pm 134$ & 2.93 & -4.27 & AMBER & $0.629 \pm 0.051$ \\
\hline GJ 887 & $\mathrm{M} 0.5 \mathrm{~V}$ & $5.78 \pm 0.03$ & 3626 & (1) & $0.503 \pm 0.025$ & -0.22 & (4) & $3797 \pm 45$ & - & - & AMBER & $0.459 \pm 0.011$ \\
\hline GJ 551 & M5.5V & $8.80 \pm 0.04$ & 3042 & (1) & $0.123 \pm 0.006$ & 0.19 & (2) & $3098 \pm 56$ & - & - & AMBER & $0.141 \pm 0.007$ \\
\hline
\end{tabular}

References: For $T_{\text {eff }}$ and $[\mathrm{Fe} / \mathrm{H}]$ :

(1) Ségransan et al. (2003); (2) Edvardsson et al. (1993); (3) CORALIE and (4) Woolf \& Wallerstein (2005).

For $v \sin i$ and $\log R_{H K}^{\prime}$ : CORALIE.

Masses for M-dwarfs are derived from Delfosse et al. (2000) while empirical relation from Xia et al. (2008) is used for K-dwarfs.

Metallicity values provided are all directly determined by spectroscopy.

two-channel combiner. For two Gaussian distributions of $V^{2}$ series, the correlation coefficient between spectral channel $k$ and a reference channel, $r$, is

$\rho_{\lambda_{r}, \lambda_{k}}=\frac{\left\langle\left(V_{\lambda_{r}}^{2}-\overline{V_{\lambda_{r}}^{2}}\right)\left(V_{\lambda_{k}}^{2}-\overline{V_{\lambda_{k}}^{2}}\right)\right\rangle}{\sqrt{\left(V_{\lambda_{r}}^{2}-\overline{V_{\lambda_{r}}^{2}}\right)^{2}\left(V_{\lambda_{k}}^{2}-\overline{V_{\lambda_{k}}^{2}}\right)^{2}}}$.

We computed $\rho_{\lambda_{r}, \lambda_{k}}$ through Monte-Carlo simulations from the mean and variance of $V_{\lambda_{r}}^{2}$ and $V_{\lambda_{k}}^{2}$. This provides a global correlation factor for a given band to estimate the amplitude of the error bar that is independent, and then to compute realistic error on the final diameter. As expected, we found that $V^{2}$ data in the same spectral band are highly correlated, so that biases the final result if correlations are not taken into account.

Error estimates on final radii obtained with AMBER mainly come from transfer function uncertainties and correlations between squared visibilities. Those factors gain in importance as the coherence time degrades.

\subsection{Data analysis}

\subsubsection{Limb-darkened diameters}

At the level of accuracy achieved on the diameter determination for K dwarfs, the discrepancy between uniform disk (UD) and limb-darkened disk (LD) is significant. Therefore, we used the nonlinear limb-darkening law describing the intensity distribution of the star disk from Claret (2000):

$I(\mu)=I(1)\left[1-\sum_{k=1}^{4} a_{k}\left(1-\mu^{k / 2}\right)\right]$,

where $I(1)$ is the specific intensity at the centre of the disk, $\mu=\cos \gamma, \gamma$ being the angle between the line of sight and the emergent intensity, and $a_{k}$ the limb-darkening coefficients. $T_{\text {eff }}$ and $\log g$ for each target are shown in Table 4, with the corresponding references. We used those parameters, added to the photometric band ( $K$ for VINCI, $H+K$ for AMBER) and microturbulence velocity (assumed to be $V_{T}=2 \mathrm{~km} \mathrm{~s}^{-1}$ ), to select the corresponding limb-darkening coefficients for the PHOENIX models.
Then, we adjusted a limb-darkened disk that is given by Davis et al. (2000) to the $V^{2}$ data:

$$
V_{\mathrm{LD}, \lambda}=\frac{\int_{0}^{1} \mathrm{~d} \mu I(\mu) \mu J_{0}\left(\pi B \theta_{\mathrm{LD}} / \lambda\left(1-\mu^{2}\right)^{1 / 2}\right)}{\int_{0}^{1} \mathrm{~d} \mu I(\mu)} .
$$

We used the same LD coefficients for $H$ and $K$ bands since the corresponding discrepancy has been evaluated at the $0.05 \%$ level on the final radius, which is negligible as compared to the error bar amplitude obtained with AMBER. Uncertainties on the same coefficients due to a slightly different $T_{\text {eff }}$ and $\log g$ can also be neglected, a $200 \mathrm{~K}$ change corresponding to a $0.01 \%$ on final radius estimate.

Table 5 lists the derived UD and LD angular diameters and their corresponding errors for both instruments. Figures 2, 3, and A.1 show $V^{2}$ data from VINCI with corresponding fitted LD models for GJ 663 A, GJ 845, and GJ 570 A, respectively. Figures 4,5 , A.2, and A.3 show $V^{2}$ data from AMBER with corresponding fitted LD models for GJ 887, GJ 166 A, GJ 551, and GJ 879, respectively.

\subsubsection{Instrument systematics}

Assessing systematics is essential for considering when reaching a few percent precision on angular diameters. We thus wanted to check for consistency between both instruments on GJ 887 and GJ 551. The results we obtained are shown in Table 5. Angular diameter determinations are consistent for both instruments. GJ 887 was observed in optimal conditions in both cases and the agreement is good at 1- $\sigma$ level, as it is for GJ 551. Bracketing each source point with two calibrators allowed better sampling of the transfer function, slightly reducing those effects as shown in Fig. 1. When good atmospheric conditions are met and a proper calibration applied, systematics on the $H$ and $K$ bands can be expected to be at the $2 \%$ level, slightly above VINCI's.

\section{Discussion}

We focussed our study on the low and very low ends of the main sequence with spectral types ranging from K0.5 to M5.5. The stars composing our sample cover a wide range of masses from 0.12 to $0.8 M_{\odot}$ - which results in different physical conditions affecting their internal structure, the heat transport, and their evolution. Their atmosphere chemistry is also strongly affected with the disappearance of true continuum to the benefit 
Table 5. Derived uniform disk and limb-darkened diameters, and stellar radii.

\begin{tabular}{llccccc}
\hline \hline Target & Instrument & $\begin{array}{c}\text { parallax } \\
{[\mathrm{mas}]}\end{array}$ & $m_{K}$ & $\begin{array}{c}\text { Limb darkening coeff. } \\
K \text { band }\end{array}$ & $\begin{array}{c}\theta_{\mathrm{UD}} \\
{[\mathrm{mas}]}\end{array}$ & $\begin{array}{c}\theta_{\mathrm{LD}} \\
{[\mathrm{mas}]}\end{array}$ \\
\hline GJ 663 A & VINCI & $168.54 \pm 0.54$ & - & {$[0.79,-0.56,0.43,-0.15]$} & $1.253 \pm 0.025$ & $1.282 \pm 0.026$ \\
GJ 166 A & AMBER & $200.62 \pm 0.23$ & $2.39 \pm 0.02(\mathrm{a})$ & {$[0.81,-0.53,0.39,-0.13]$} & $1.405 \pm 0.038$ & $1.437 \pm 0.039$ \\
GJ 570 A & VINCI & $171.22 \pm 0.94$ & $3.15 \pm 0.02(\mathrm{a})$ & {$[0.86,-0.52,0.37,-0.12]$} & $1.147 \pm 0.029$ & $1.177 \pm 0.030$ \\
GJ 845 & VINCI & $276.06 \pm 0.28$ & $2.18 \pm 0.02(\mathrm{~b})$ & {$[0.86,-0.53,0.38,-0.13]$} & $1.834 \pm 0.016$ & $1.881 \pm 0.017$ \\
GJ 879 & AMBER & $131.42 \pm 0.62$ & $3.95 \pm 0.08(\mathrm{a})$ & {$[0.86,-0.53,0.38,-0.13]$} & $0.750 \pm 0.066$ & $0.769 \pm 0.067$ \\
GJ 887 & AMBER & $305.26 \pm 0.70$ & $3.36 \pm 0.02(\mathrm{a})$ & {$[1.61,-2.35,2.00,-0.68]$} & $1.284 \pm 0.031$ & $1.304 \pm 0.032$ \\
GJ 551 & AMBER & $771.64 \pm 2.60$ & $4.38 \pm 0.03$ (c) & {$[1.94,-2.80,2.39,-0.81]$} & $0.990 \pm 0.050$ & $1.011 \pm 0.052$ \\
\hline
\end{tabular}

References: (a) Morel \& Magnenat (1978); (b) Mould \& Hyland (1976); (c) 2MASS (Cutri et al. 2003) and (d) Ségransan et al. (2003). Parallaxes are from Hipparcos.

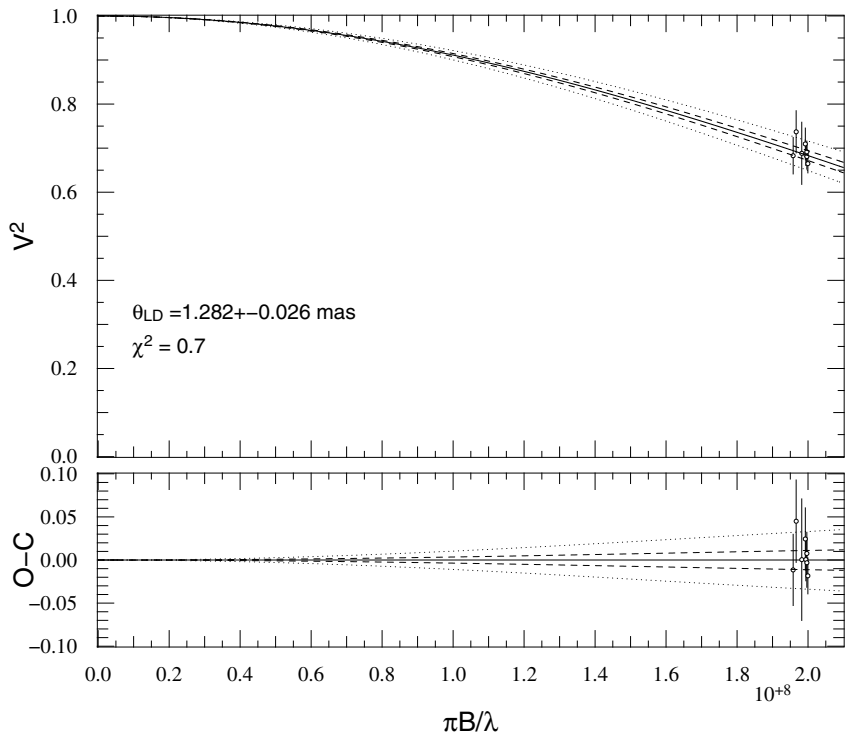

Fig. 2. Calibrated squared visibilities from VINCI and the best-fit LD disk model (solid) for GJ663A vs. spatial frequencies. 1- $\sigma$ (dash) and 3- $\sigma($ dot $)$ uncertainties are also indicated.

of a complex and a high gravity stellar atmosphere with strong molecular absorptions bands. Metallicity and activity also play a role, although we expect it to be a second order effect, at least in the near-infrared. In the following sections, we discuss the implications and constraints brought by our measurements on stellar physics modelling.

\subsection{Luminosity-radius relationship}

We first chose to compare our results to Baraffe et al. (1998) models, in a luminosity-radius diagram that corresponds to the observables. Indeed, it has the advantage of avoiding the inclusion of the mass-luminosity (hereafter ML) relationship, for which reliability regarding $\mathrm{K}$ dwarfs has not been demonstrated yet. Figure 6 shows our VLTI results. Different sets of models are overplotted, ie. for an age of $5 \mathrm{Gyr}$, featuring different mixing lengths (Böhm-Vitense 1958), $L_{\text {mix }}=\alpha H_{\mathrm{P}}$ expressed in pressure scale height $H_{\mathrm{P}}$, which allows assessment of the convective efficiency, as well as two distinct metallicities: $[\mathrm{M} / \mathrm{H}]=0$ and $[\mathrm{M} / \mathrm{H}]=-0.5$.

Baraffe et al. (1998) models are in excellent agreement with our observations in the very low-mass part of the luminosityradius diagram. The radius determined for Proxima (GJ 551) is perfectly reproduced by theory. In this part of the relation, stars

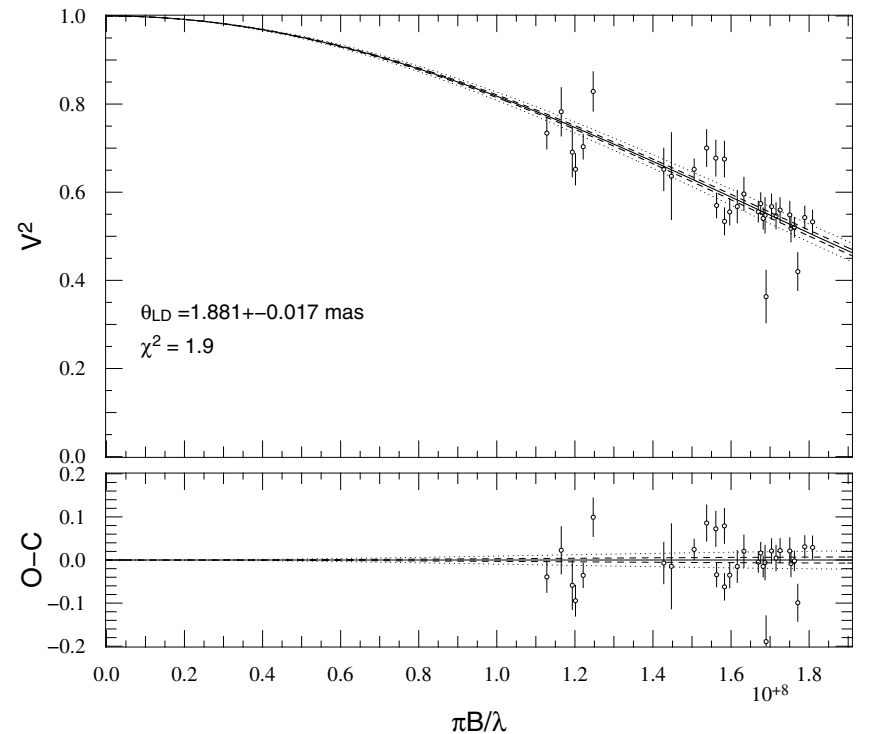

Fig. 3. Calibrated squared visibilities from VINCI and best-fit LD disk model (solid) for GJ845 vs. spatial frequencies. 1- $\sigma$ (dash) and 3- $\sigma$ (dot) uncertainties are also indicated.

are fully convective, which greatly simplifies the modelling of heat transport; therefore, our result validates the equation of state used by Baraffe et al. (1998). GJ 887 is an early type M dwarf, located slightly above the boundary of this class of objects. The radius determined for GJ 887 is also in perfect agreement with model predictions. Other measurements from the literature confirm that stellar interior physics for this mass range have been mastered.

At the time of writing this paper, there are relatively few radii measurements that would allow a discussion in the $0.5-0.75 M_{\odot}$ region. The $61 \mathrm{Cyg} \mathrm{A}$ and $\mathrm{B}$ radii have been recently determined by Kervella et al. (2008) and are also reproduced by theory. We note that Berger et al. (2006) published 6 radii measured with the CHARA array in this part of the diagram. The authors claim discrepancies with models at the 2 to $3 \sigma$ level. Such large departures from theory have not been observed by other studies. One may note, however, that 5 of the 6 stars measured by Berger et al. (2006) have inflated radii. Those stars were measured with the instrument "CHARA-Classic", a recombiner that does not include a single-mode filtering. Such measurements are prone to systematic calibration errors and indeed, the one star (GJ 15 A) that they measured with "CHARA-FLUOR" (instrument with single-mode filtering) is in excellent agreement with the models. 


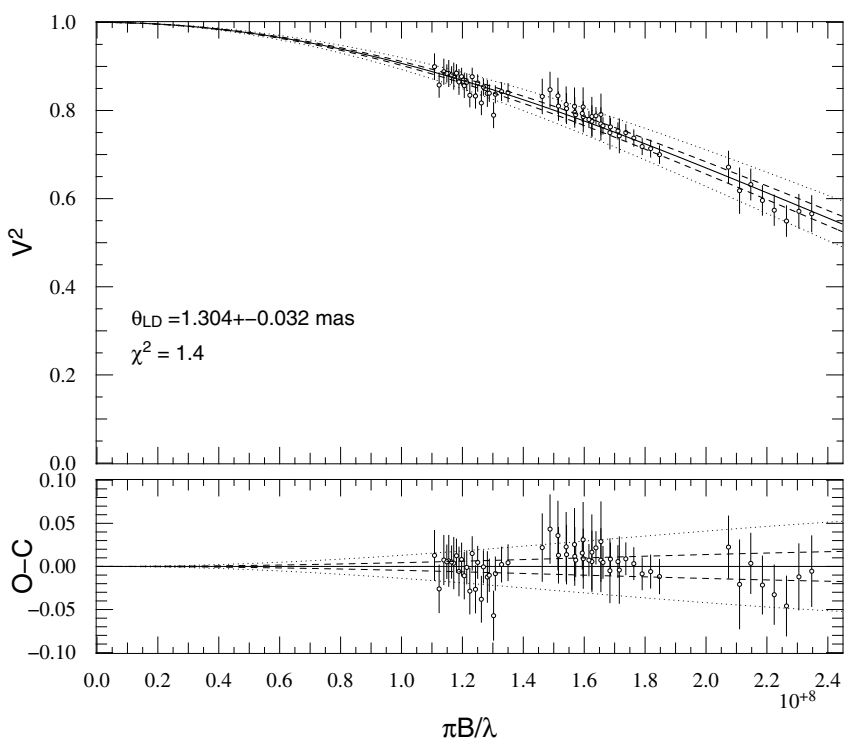

Fig. 4. Calibrated squared visibilities from AMBER (low-resolution mode) and best-fit LD disk model (solid) for GJ887 vs. spatial frequencies. 1- $\sigma$ (dash) and 3- $\sigma$ (dot) uncertainties are also indicated. Error-bar amplitudes include both correlated and non-correlated errors.

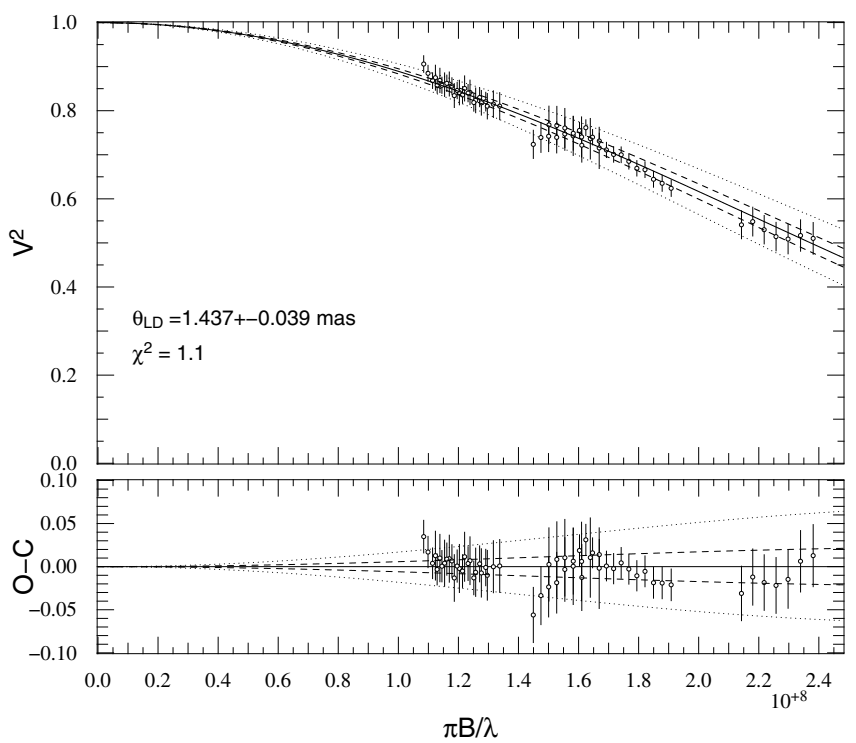

Fig. 5. Calibrated squared visibilities from AMBER (low-resolution mode) and best-fit LD disk model (solid) for GJ166A vs. spatial frequencies. 1- $\sigma$ (dash) and 3- $\sigma$ (dot) uncertainties are also indicated. Error-bar amplitudes include both correlated and non-correlated errors.

Although a possible explanation, we note that such instrumental effect is expected to result in a uniform dispersion. We decided, however, not to include those results in this discussion.

GJ 205 radius, as measured with VINCI, is about $15 \%$ above models. Radial-velocity measurements on this object have not revealed any massive (heavier than a Saturn-mass) companion (Bonfils, priv. comm.) that would have induced a lower interferometric visibility, thus a larger radius. Moreover, GJ 205 has not been reported to show significant activity (López-Morales 2007). Nevertheless, this star is more luminous than other known objects belonging to the same spectral class and is probably inflated.

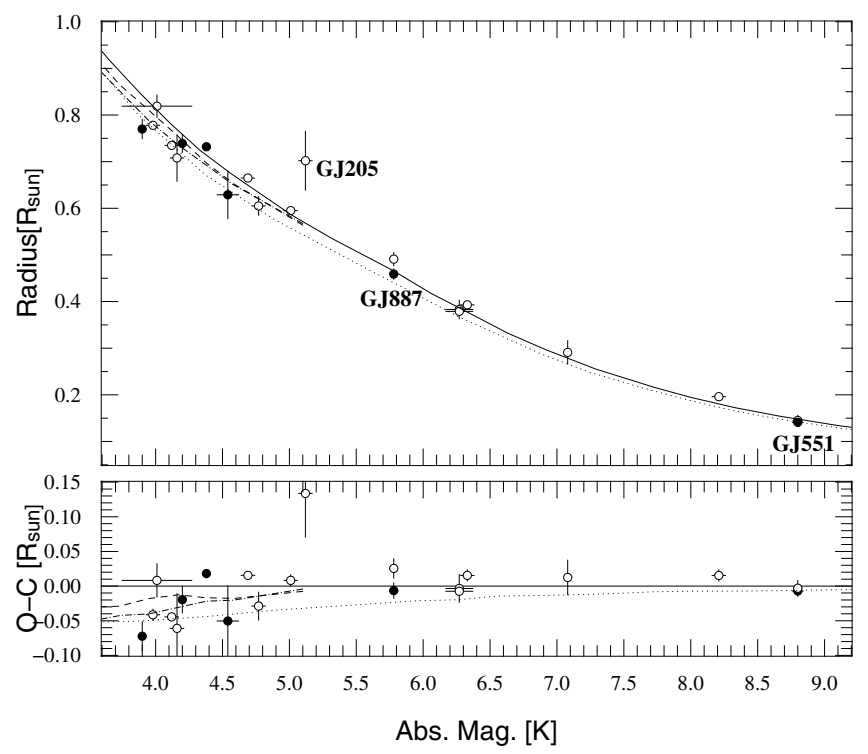

Fig. 6. Luminosity-radius relationship - Single stars radii vs. absolute $\mathrm{K}$ magnitudes superimposed on $5 \mathrm{Gyr}$ isochrones theoretical models (Baraffe et al. 1998). Our results from AMBER and VINCI are shown as filled circles. We have also included other stellar radii from the literature determined by interferometry: Berger et al. (2006) for GJ 15 A, Boyajian et al. (2008), di Folco et al. (2007), Kervella et al. (2008), Ségransan et al. (2003), and Lane et al. (2001) as empty circles. Only radii measurements with an accuracy better than $10 \%$ are displayed. Different models for $5 \mathrm{Gyr}$ isochrones are also shown: solar metallicity with $L_{\text {mix }}=1.0 H_{\mathrm{P}}$ (solid), $L_{\text {mix }}=1.5 H_{\mathrm{P}}($ dash $)$, and $L_{\text {mix }}=1.9 H_{\mathrm{P}}$ (dashdot), as well as a metal-deficient, $[\mathrm{M} / \mathrm{H}]=-0.5$ model with $L_{\text {mix }}=1.0 H_{\mathrm{P}}(\mathrm{dot})$. GJ 205, GJ 887, and GJ 551 that appear in the discussion are labelled.

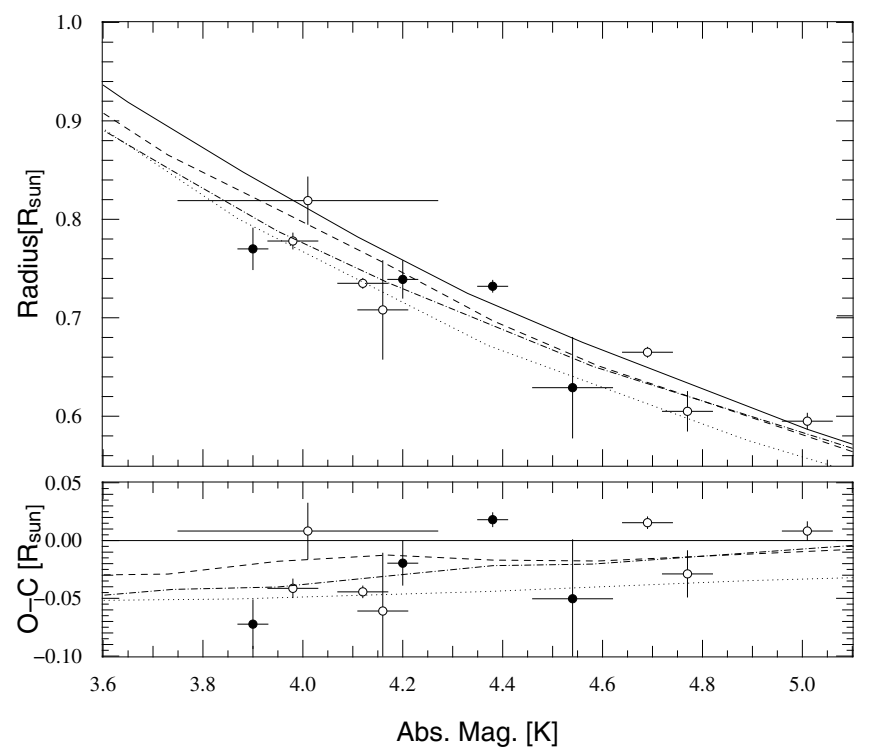

Fig. 7. Luminosity-radius relationship - same as Fig. 6, zoomed on the upper part of the diagram.

In the upper part of the luminosity-radius relationship, models reproduce the observations provided that larger mixing length parameter $\alpha$ are used (such as $L_{\text {mix }}=1.5 H_{\mathrm{P}}$ and the solar $L_{\text {mix }}=1.9 H_{\mathrm{P}}$ ). This part of the relationship may be used to calibrate $L_{\text {mix }}$, provided accurate observational radii and magnitude determination are available.

Figure 7 displays a zoom on this area of the relation. 


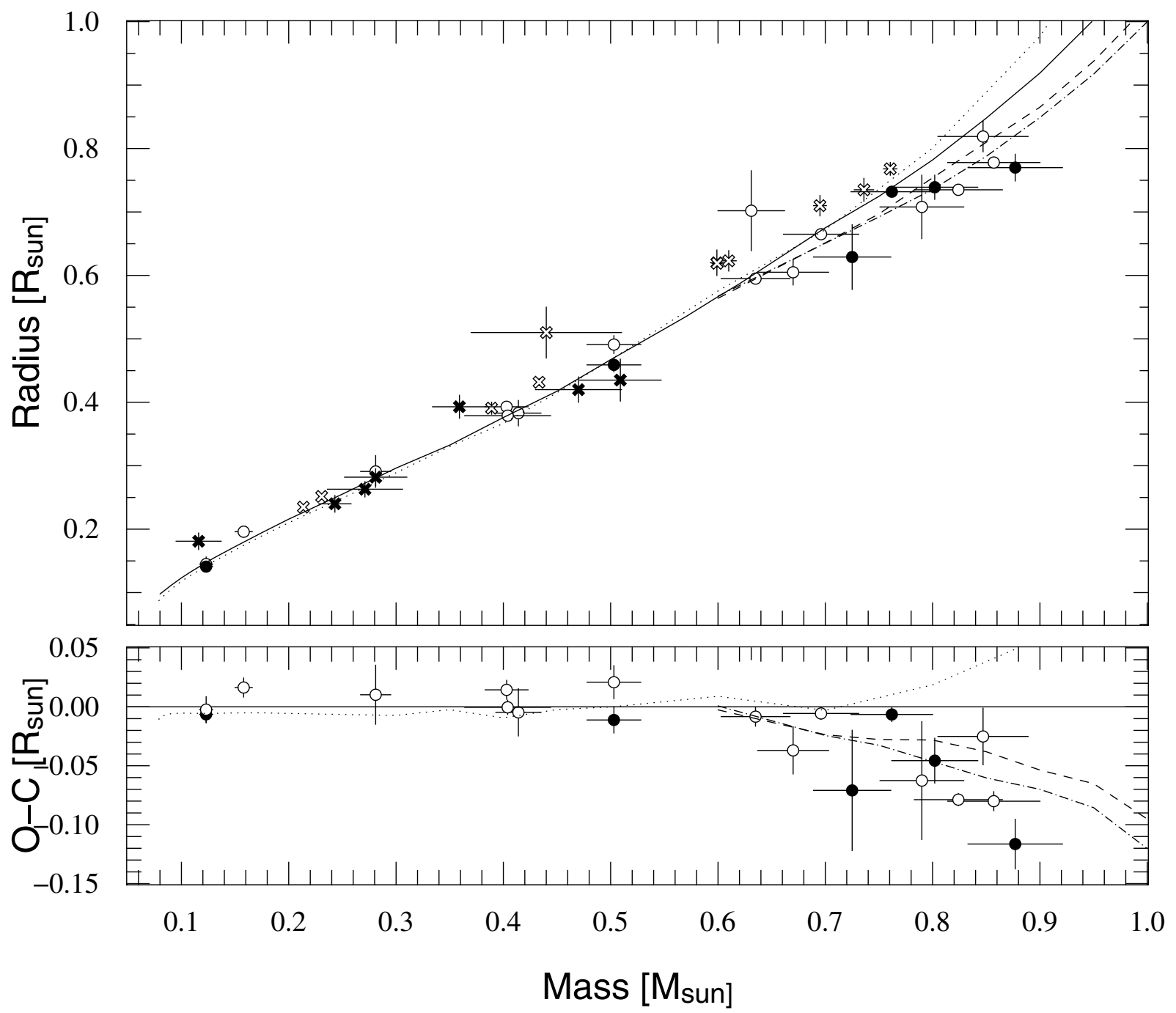

Fig. 8. Mass-radius relationship - masses and radii superimposed on 5 Gyr isochrones theoretical models (Baraffe et al. 1998). Our results appear as filled circles. Other long baseline interferometry measurements come from PTI (Lane et al. 2001), VLTI (Ségransan et al. 2003) and CHARAFLUOR: Boyajian et al. (2008), di Folco et al. (2007), Kervella et al. (2008) and Berger et al. (2006) for GJ15A, all as empty circles. Solar metallicity with $L_{\text {mix }}=1.0 H_{\mathrm{P}}$ (solid), $L_{\text {mix }}=1.5 H_{\mathrm{P}}$ (dash) and $L_{\text {mix }}=1.9 H_{\mathrm{P}}$ (dashdot) are shown as well as a metal deficient, [M/H] $=-0.5 \mathrm{model}$ with $L_{\text {mix }}=1.0 H_{\mathrm{P}}(\mathrm{dot})$. Only radii measurements better than $10 \%$ are displayed. Solar-neighbourhood eclipsing binary measurements are represented as empty crosses, while OGLE-T transiting binaries are represented in filled crosses. Only residuals from long-baseline interferometry results are displayed.

Unfortunately, GJ 663 A does not appear in the tables or in the graphs because of the lack of $K$ magnitude measurements. Its measured radius is, however, shown in Table 5 for completeness. It should be noted that some efforts are needed to obtain accurate near-infrared photometry of nearby $\mathrm{K}$ dwarfs to tighten the mass-luminosity relation and therefore better constrain theoretical models in the upper part of the luminosityradius relationship.

\subsection{Mass-radius relationship}

The translation of our direct measurements into a mass-radius diagram requires the use of an empirical ML relationship. We used the relation determined by Delfosse et al. (2000) to compute masses for GJ 887 and GJ 551, and a recent one by Xia et al. (2008) for the 0.7 to $1.0 M_{\odot}$ range. This study is based on Henry $\&$ McCarthy (1993). The ML relationship for stars below $0.6 M_{\odot}$ is built on a large number of accurate masses and luminosities (Ségransan et al. 2000) and exhibits very low dispersion in near-infrared. In this part of the mass-luminosity relationship, models reproduce the observations fairly well, indicating that both atmosphere and interior physics of very low-mass have been mastered. The empirical ML relationship above $0.6 M_{\odot}$ shows a much larger dispersion than for M dwarfs, which is related to the modest accuracy of the masses in this mass range. The derivation of masses from absolute magnitude is therefore less accurate than for the lower part of the relation, so we adopted an arbitrary error of 5\% on mass determination. Those results appear in Table 5. Figure 8 shows our results in a massradius (MR) diagram with results from other studies. Five Gyr model isochrones from Baraffe et al. (1998) are represented for different mixing lengths and stellar metallicity.

\subsection{Stellar properties}

\subsubsection{Effective temperature}

Angular diameter measurements associated with accurate parallax and Johnson photometry allow the star's effective 
temperature to be derived. UV to near IR photometry were obtained from the literature, mostly from Morel \& Magnenat (1978). Visual and near-infrared photometry appear in Table B.2. We derived effective temperatures by inverting the surfacebrightness empirical relations calibrated by Kervella et al. (2004b). The $T_{\text {eff }}$ values determined through this method are in good agreement with $T_{\text {eff }}$ determined by spectroscopy, and both appear in Table 5.

\subsubsection{Metallicity}

Metallicity effects have been mentioned by Berger et al. (2006) to explain the difference between four of his measurements and solar metallicity models. Indeed, the authors claim that missing opacity sources in the models, such as TiO, would explain the models' underestimation of stellar radii for some M dwarfs. López-Morales (2007) recently studied the correlation between magnetic activity, metallicity and low-mass stars radii. Based on Berger et al. (2006) measurements, she reach the same conclusion. However, no other instrument (PTI, VINCI, CHARA-FLUOR or AMBER) could confirm this hypothesis except for GJ 205 (VINCI) as explained in Sect. 3.1. Without the "CHARA-Classic" measurements by Berger et al. (2006), the metallicity-radius diagram for single stars (Fig. 9) no longer shows this correlation.

\subsubsection{Activity}

Close-in eclipsing binaries (EB), for which accurate masses and radii have been measured by several authors such as Torres \& Ribas (2002), show significant discrepancies with stellar models (see e.g. Ribas et al. 2008). Recently, Chabrier et al. (2007) explained those discrepancies by invoking the reduced convective efficiency and starspots coverage of eclipsing binaries. The difference is only observed in mass-radius diagrams (and not in the luminosity-radius diagram) because the slightly lower effective temperature of EB is compensated for by a larger radius, only slightly changing the luminosity. This explanation is only meaningful for EB with periods of a few days implying heavy tidal effects, orbital synchronization, hence an enhanced activity. This trend is shown in Fig. 8.

However, the same arguments cannot be used for single stars. Rotational velocity is an excellent hint of stellar activity. While low-mass and very low-mass EB are routinely characterised by $v \sin i$ between $7.11 \mathrm{~km} \mathrm{~s}^{-1}$ (CU Cnc B, Ribas 2003) and $129.5 \mathrm{~km} \mathrm{~s}^{-1}$ (OGLE BW3 V38A, Maceroni \& Montalbán 2004), single stars rarely exceed $3 \mathrm{~km} \mathrm{~s}^{-1}$. We assessed stellar activity for our targets thanks to CORALIE spectra, and it indeed appears that all of them do not show any activity comparable to EB ones. Corresponding $\log R_{H K}^{\prime}$ and $v \sin i$ appear in Table 5. Furthermore, activity cannot explain the radii discrepancies reported by Berger et al. (2006), since none of those single stars in their sample show high activity levels (López-Morales 2007).

Activity cannot be claimed as the source of deviation in the upper part of the mass-radius relationship for single stars. However, radii of single inactive $\mathrm{M}$ dwarfs measured by interferometry are in excellent agreement with models from Baraffe et al. (1998). Thus, the discrepancies pointed out by Torres \& Ribas (2002) and Ribas (2003) only concern fast-rotating stars, confirming that rotation strongly affects the internal structure of those objects.

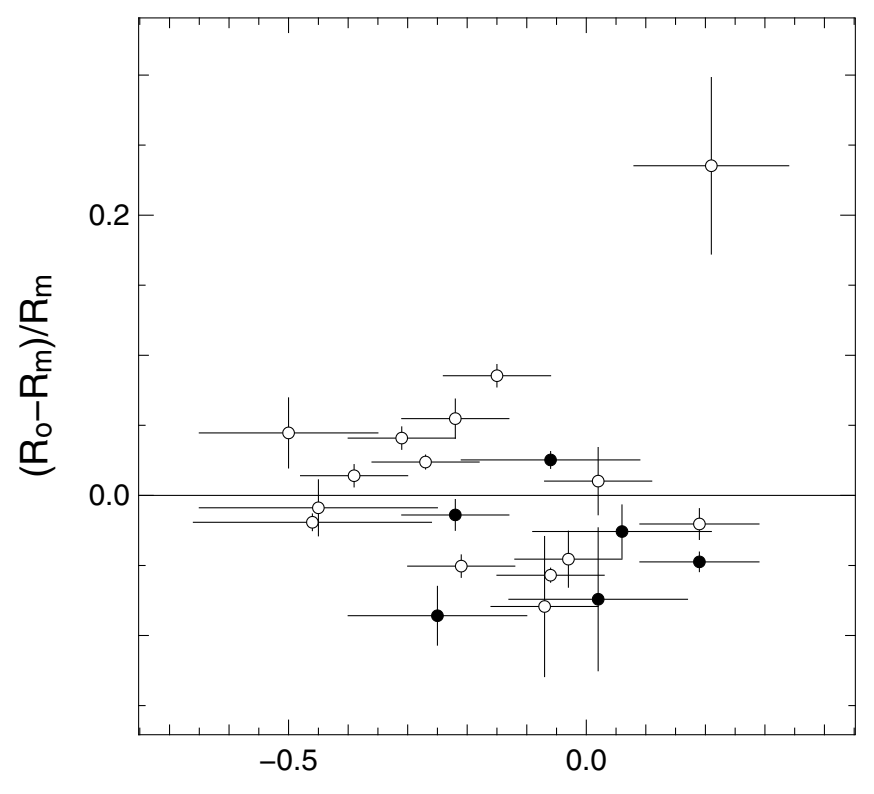

$[\mathrm{Fe} / \mathrm{H}] \mathrm{dex}$

Fig. 9. Fractional deviation of single stars radii derived by interferometry from a $5-\mathrm{Gyr}, L_{\mathrm{mix}}=1.0 H_{\mathrm{P}}$ model (Baraffe et al. 1998) vs. stellar metallicity.

\section{Conclusion}

These new results obtained at the VLTI with its near-infrared instruments, VINCI and AMBER, allow the mass-radius relationship for low and very low mass stars to be better constrained. We have shown that AMBER is now able to achieve high-quality absolute visibility measurements provided that at least 3 calibrator stars are observed and that a careful data reduction and analysis is conducted. Using VINCI as a benchmark, those results are also shown to be reliable, even if the proposed approach requires a time-consuming observing strategy. Assessment of potential systematics, as well as realistic error bars estimates, have been crucial to comparing our results with models in a meaningful way.

Models are in good agreement with the observations, confirming a correct understanding of the underlying physics of low and very low mass stars. The very low-mass regime is almost adiabatic and thus constraints the equation of state. A mixing length parameter $\alpha$ of 1.0 leads to a progressive underestimation of radii for early $\mathrm{K}$ dwarfs, that are better represented by a solar mixing length parameter $(\alpha=1.9)$, as expected.

Acknowledgements. We are very grateful to Guy Perrin and Florentin Millour for their advice about AMBER data reduction, as well to Stanislav Stefl and Carla Gil for their work as VLTI night astronomers. B.-O.D. also would like to thank Fabien Malbet and Guy Perrin for having organised a school devoted to interferometry at Goutelas in 2006. Many thanks also to France Allard and Corinne Charbonnel for fruitful discussions about very low-mass stars atmospheres and interior physics. We thank the anonymous referee for constructive comments on the manuscript. This work benefits from the support of the Fonds National Suisse de la Recherche Scientifique. This study made use of amdlib 2.2 developed by the Jean-Marie Mariotti Centre, supported by INSU (CNRS and Ministère de la recherche, France). This research made use of the SIMBAD database operated by the CDS, Strasbourg, France. This publication makes use of data products from the Two Micron All Sky Survey, which is a joint project of the University of Massachusetts and the Infrared Processing and Analysis Center/California Institute of Technology, funded by the National Aeronautics and Space Administration and the National Science Foundation. 


\section{Appendix A: Additional Material - Figures}

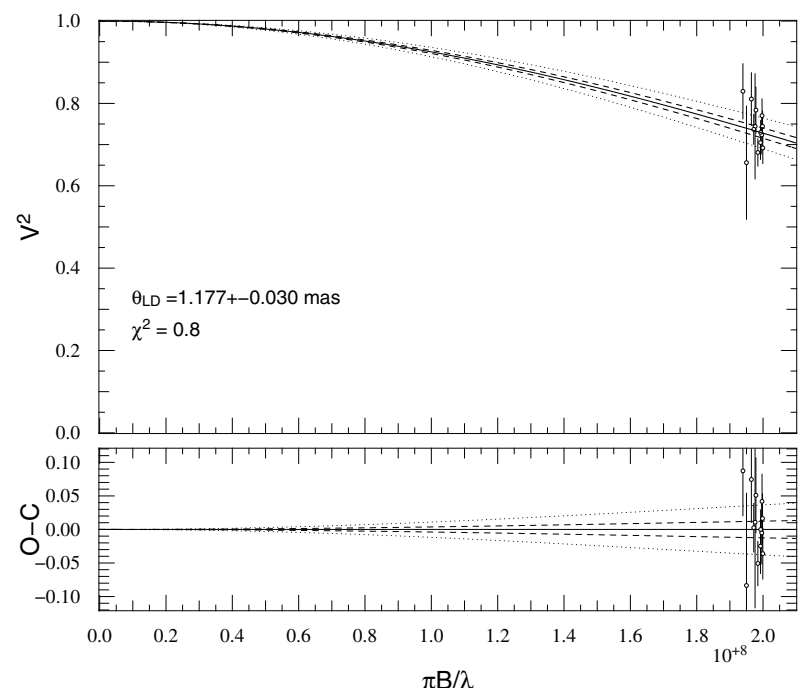

Fig. A.1. Calibrated squared visibilities from VINCI and best-fit LD disk model (solid) for GJ570A vs. spatial frequencies. 1- $\sigma$ (dash) and 3- $\sigma$ (dot) uncertainties are also indicated.

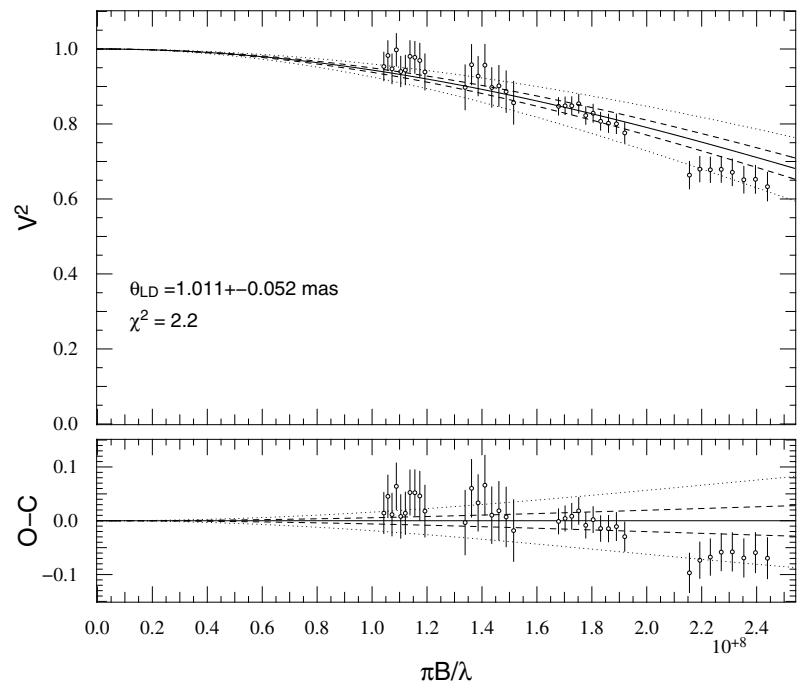

Fig. A.2. Calibrated squared visibilities from AMBER (low-resolution mode) and best-fit LD disk model (solid) for GJ551 vs. spatial frequencies. 1- $\sigma$ (dash) and 3- $\sigma$ (dot) uncertainties are also indicated. Error bars amplitudes include both correlated and non-correlated errors.

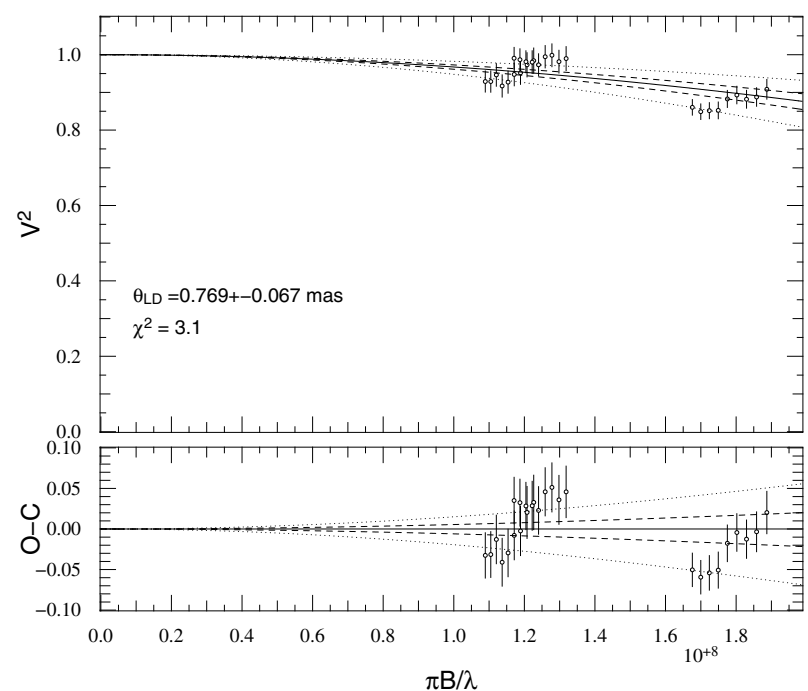

Fig. A.3. Calibrated squared visibilities from AMBER (low-resolution mode) and best-fit LD disk model (solid) for GJ879 vs. spatial frequencies. 1- $\sigma$ (dash) and 3- $\sigma$ (dot) uncertainties are also indicated. Error bars amplitudes include both correlated and non-correlated errors. 


\section{Appendix B: Additional Material - Tables}

Table B.1. Online material - Angular diameters and linear radii of single low and very-low mass stars from the literature, respectively expressed in mas and sun radii.

\begin{tabular}{llrclcccc}
\hline \hline Star & Spect. & {$[\mathrm{Fe} / \mathrm{H}]$} & Ref.[Fe/H] & Instr. & $\theta_{\mathrm{UD}}$ & $\theta_{\mathrm{LD}}$ & $R_{\text {lin }}$ & Ref. $_{\text {interf. }}$ \\
\hline$\sigma$ Dra & K0V & -0.21 & (a) & CHARA & $1.224 \pm 0.011$ & $1.254 \pm 0.012$ & $0.778 \pm 0.008$ & $(4)$ \\
HR 511 & K0V & 0.02 & (a) & CHARA & $0.747 \pm 0.021$ & $0.763 \pm 0.021$ & $0.819 \pm 0.024$ & $(4)$ \\
$\epsilon$ Eri & K2V & -0.06 & (a) & VINCI & $2.093 \pm 0.029$ & $2.148 \pm 0.029$ & $0.735 \pm 0.005$ & $(1)$ \\
GJ 105 A & K3V & -0.07 & (a) & PTI & $0.914 \pm 0.07$ & $0.936 \pm 0.07$ & $0.708 \pm 0.050$ & $(2)$ \\
61 Cyg A & K5V & -0.27 & (a) & CHARA & & $1.775 \pm 0.013$ & $0.665 \pm 0.005$ & $(5)$ \\
GJ 380 & K7V & -0.03 & (a) & PTI & $1.268 \pm 0.04$ & $1.155 \pm 0.04$ & $0.605 \pm 0.020$ & $(2)$ \\
61 Cyg B & K7V & -0.39 & (a) & CHARA & & $1.581 \pm 0.022$ & $0.595 \pm 0.008$ & $(5)$ \\
GJ 191 & M1V & -0.50 & (b) & VINCI & $0.681 \pm 0.06$ & $0.692 \pm 0.06$ & $0.291 \pm 0.025$ & $(3)$ \\
GJ 887 & M0.5V & -0.22 & (c) & VINCI & $1.366 \pm 0.04$ & $1.388 \pm 0.04$ & $0.491 \pm 0.014$ & $(3)$ \\
GJ 205 & M1.5V & 0.21 & (c) & VINCI & $1.124 \pm 0.11$ & $1.149 \pm 0.11$ & $0.702 \pm 0.063$ & $(3)$ \\
GJ 15 A & M2V & -0.45 & (d) & PTI & $0.976 \pm 0.016$ & $0.988 \pm 0.016$ & $0.379 \pm 0.006$ & $(6)$ \\
GJ 411 & M1.5V & -0.31 & (a) & PTI & $1.413 \pm 0.03$ & $1.436 \pm 0.03$ & $0.393 \pm 0.008$ & $(2)$ \\
GJ 699 & M4Ve & -0.15 & (a) & PTI & $0.987 \pm 0.04$ & $1.004 \pm 0.04$ & $0.196 \pm 0.008$ & $(2)$ \\
GJ 551 & M5.5V & 0.19 & (f) & VINCI & $1.023 \pm 0.08$ & $1.044 \pm 0.08$ & $0.145 \pm 0.011$ & $(3)$ \\
\hline
\end{tabular}

Ref. for [Fe/H]: (a) Soubiran et al. (2008); (b) Mould \& Hyland (1976); (c) Woolf \& Wallerstein (2005); (d) Bonfils et al. (2005); (e) Edvardsson et al. (1993).

Ref. for interferometric measurements: (1) di Folco et al. (2007); (2) Lane et al. (2001); (3) Ségransan et al. (2003); (4) Boyajian et al. (2008); (5) Kervella et al. (2008); (6) Berger et al. (2006).

Table B.2. Apparent magnitudes of stars included in this study. Uncertainty adopted for each apparent magnitude value is given in superscript.

\begin{tabular}{|c|c|c|c|c|c|c|c|c|c|}
\hline Star & $m_{U}^{a}$ & $m_{B}^{b}$ & $m_{V}^{b}$ & $m_{R}^{c}$ & $m_{I}^{c}$ & $m_{J}^{d}$ & $m_{H}^{d}$ & $m_{K}{ }^{d}$ & $m_{L}^{d}$ \\
\hline GJ 166 A & $5.69^{0.02}$ & $5.25^{0.02}$ & $4.43^{0.02}$ & $3.72^{0.01}$ & $3.27^{0.01}$ & $2.91^{0.03}$ & $2.46^{0.01}$ & $2.39^{0.02}$ & $2.30^{0.02}$ \\
\hline GJ 663 A & $5.67^{0.02}$ & $5.18^{0.02}$ & $4.32^{0.02}$ & $3.62^{0.02}$ & $3.18^{0.02}$ & & & & \\
\hline GJ $570 \mathrm{~A}$ & $7.88^{0.02}$ & $6.82^{0.02}$ & $5.71^{0.02}$ & $4.72^{0.02}$ & $4.18^{0.02}$ & $3.82^{0.02}$ & $3.27^{0.02}$ & $3.15^{0.02}$ & $3.11^{0.02}$ \\
\hline GJ 845 & $6.74^{0.02}$ & $5.75^{0.02}$ & $4.69^{0.02}$ & $3.81^{0.02}$ & $3.25^{0.02}$ & $2.83^{0.02}$ & $2.30^{0.02}$ & $2.18^{0.02}$ & $2.12^{0.02}$ \\
\hline GJ 879 & $8.61^{0.02}$ & $7.59^{0.02}$ & $6.49^{0.02}$ & $5.54^{0.02}$ & $4.95^{0.02}$ & $4.53^{0.04}$ & $4.00^{0.07}$ & $3.95^{0.08}$ & \\
\hline GJ 887 & $9.99^{0.02}$ & $8.83^{0.02}$ & $7.35^{0.02}$ & & & $4.20^{0.02}$ & $3.60^{0.02}$ & $3.36^{0.02}$ & $3.20^{0.02}$ \\
\hline GJ 551 & $14.56^{0.02}$ & $13.02^{0.02}$ & $11.05^{0.02}$ & $8.68^{0.02}$ & $6.42^{0.02}$ & $5.33^{0.02}$ & $4.73^{0.02}$ & $4.36^{0.03}$ & $4.04^{0.02}$ \\
\hline
\end{tabular}

References: ${ }^{a}$ Morel \& Magnenat (1978); ${ }^{b}$ Morel \& Magnenat (1978); van Leeuwen (2007); ${ }^{c}$ Morel \& Magnenat (1978); Ducati (2002); ${ }^{d}$ Morel \& Magnenat (1978), Glass (1974), Mould \& Hyland (1976), Ducati (2002), Cutri et al. (2003).

\section{References}

Baraffe, I., Chabrier, G., Allard, F., \& Hauschildt, P. H. 1998, A\&A, 337, 403 Berger, D. H., Gies, D. R., McAlister, H. A., et al. 2006, Am. Astron. Soc., AJ, 644,475 ,

Böhm-Vitense, E. 1958, Zeitschrift fur Astrophysik, 46, 108

Bonfils, X., Delfosse, X., Udry, S., et al. 2005, A\&A, 442, 635

Bordé, P., Coudé du Foresto, V., Chagnon, G., \& Perrin, G. 2002, A\&A, 393, 183

Boyajian, T. S., McAlister, H. A., Baines, E. K., et al. 2008, ApJ, 683, 424

Chabrier, G., Gallardo, J., \& Baraffe, I. 2007, A\&A, 472, L17

Claret, A. 2000, A\&A, 363, 1081

Coude Du Foresto, V., Perrin, G., Ruilier, C., et al. 1998, in Presented at the Society of Photo-Optical Instrumentation Engineers (SPIE) Conf. ed. R. D. Reasenberg, Conf. Ser., 3350, 856

Cutri, R. M., Skrutskie, M. F., van Dyk, S., et al. 2003, 2MASS All Sky Catalog of point sources, The IRSA 2MASS All-Sky Point Source Catalog, NASA/IPAC Infrared Science Archive, http://irsa.ipac.caltech. edu/applications/Gator/

Davis, J., Tango, W. J., \& Booth, A. J. 2000, Monthly Notices RAS, R. Astron. Soc., 318,387

Delfosse, X., Forveille, T., Ségransan, D., et al. 2000, A\&A, 364, 217

di Folco, E., Absil, O., Augereau, J.-C., et al. 2007, A\&A, 475, 243

Ducati, J. R. 2002, VizieR Online Data Catalog, 2237, 0

Edvardsson, B., Andersen, J., Gustafsson, B., et al. 1993, A\&A, 275, 101

Glass, I. S. 1974, Monthly Notes of the Astronomical Society of South Africa, 33,53

Henry, T. J., \& McCarthy, D. W. 1993, AJ, 106, 773

Kervella, P., Coude du Foresto, V., Glindemann, A., \& Hofmann, R. 2000, SPIE Conf. 4006, ed. P. Léna, \& A. Quirrenbach, 31

Kervella, P., Ségransan, D., \& du Foresto, V. C. 2004a, A\&A, 425, 1161
Kervella, P., Thévenin, F., DiFolco, E., \& Ségransan, D. 2004b, A\&A, 426, 297 Kervella, P., Mérand, A., Pichon, B., et al. 2008, A\&A, 488, 667

Lane, B. F., Boden, A. F., \& Kulkarni, S. R. 2001, Am. Astron. Soc., AJ, 551, L81

Le Bouquin, J.-B., Abuter, R., Bauvir, B., et al. 2008, in SPIE Conf. Ser., 7013 López-Morales, M. 2007, AJ, 660, 732

Maceroni, C., \& Montalbán, J. 2004, A\&A, 426, 577

Malbet, F., Duvert, G., Chelli, A., \& Kern, P. 2008, AMBER Task Force February 2008 run report, arXiv e-prints

Merand, A., Borde, P., \& Foresto, V. C. D. 2004, New Frontiers in Stellar Interferometry, Proc. SPIE 5491, 1185

Morel, M., \& Magnenat, P. 1978, A\&AS, 34, 477

Mould, J. R., \& Hyland, A. R. 1976, ApJ, 208, 399

Perrin, G. 2003, A\&A, 400, 1173

Petrov, R. G., Malbet, F., Weigelt, G., et al. 2007, A\&A, 464, 1

Ribas, I. 2003, A\&A, 398, 239

Ribas, I., Morales, J. C., Jordi, C., et al. 2008, Mem. Soc. Astron. Ital., 79, 562 Ségransan, D., Forveille, T., Millan-Gabet, C. P. R., \& Traub, W. A. 1999, in Working on the Fringe: Optical and IR Interferometry from Ground and Space, ed. S. Unwin, \& R. Stachnik, ASP Conf. Ser., 194, 290

Ségransan, D., Delfosse, X., Forveille, T., et al. 2000, A\&A, 364, 665

Ségransan, D., Kervella, P., Forveille, T., \& Queloz, D. 2003, A\&A, 397, L5

Soubiran, C., Bienaymé, O., Mishenina, T. V., \& Kovtyukh, V. V. 2008, A\&A, 480, 91

Tatulli, E., Millour, F., Chelli, A., et al. 2007, A\&A, 464, 29

Torres, G., \& Ribas, I. 2002, ApJ, 567, 1140

van Leeuwen, F., ed. 2007, Hipparcos, the New Reduction of the Raw Data, Astrophys. Space Sci. Library, 250,

Woolf, V. M., \& Wallerstein, G. 2005, MNRAS, 356, 963

Xia, F., Ren, S., \& Fu, Y. 2008, Astrophys. Space Sci. (Springer Science+ Business Media B.V.), 314, 51 